attached $X$-chromosomes obtained by us gave a progeny, part of which in fact contained a ring chromosome (Fig. 1 (right hand), Stern's culture $\left.x y^{\prime} y^{\prime \prime}\right)$.

The frequency of single crossing-over (number of distal recombinations) had at the same time not decreased. Here, too, in all the one hundred per cent of ovocytes, the same as under normal conditions, the $X$-chromosome undergoes crossing-over and forms at least one chiasma.
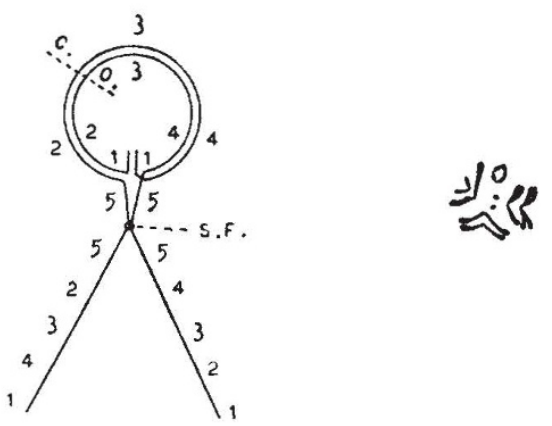

FIG. 1.

A study of double crossing-over in a similar heterozygous inversion $s c^{8}$ (non-attached $X$-chromosomes) has shown that the difference between the frequency of double cross-overs in a heterozygous inversion $s c^{8}$ and that of double crossing-over in a homozygous one, may be explained by the occurrence of new regional differences during ring conjugation. The increased percentage of non-disjunctional males and females which arise in a heterozygous inversion are a result of double crossing-over. Experiments show that the increase of double crossing-over in a heterozygous inversion causes an increase of the number of exceptional individuals. The presence of the latter cannot therefore prove the disturbance of chromosome conjugation.

The data obtained by us in this work indicate that in a heterozygous inversion of Drosophila, chromosome conjugation occurs in the same way as has been described by McClintock for Zea Mays. There the forces of attraction of homologous loci which bend the chromosome into a ring cause complete conjugation, notwithstanding the presence of an inversion in one of the chromosomes.

The existence of a Drosophila stock with attached $X$-chromosomes where ring chromosomes are permanently formed, is a new cytogenetic proof of Morgan's theory of crossing-over.

B. N. SIDorov.

N. N. Sokolov.

I. E. Trofimov.

Department of Genetics,

Institute of Experimental Biology, Moscow.

May 7.

\section{Cosmic Ray Bursts in Liquid Dielectrics}

Bursts of ionisation produced by cosmic rays have been studied up to now in large ionisation chambers filled with a gas at high pressure. We found it advantageous to use for this purpose ionisation chambers filled with a very carefully purified liquid dielectric.

A series of preliminary observations was made with the use of a cylindrical condenser having a capacity of nearly 1 litre containing hexane $\left(\mathrm{C}_{6} \mathrm{H}_{14}\right)$; the interior armature was an empty cylindrical vessel. The conductivity of the hexane was as low as $2 \cdot 3 \times$ $10^{-19} \Omega^{-1} \mathrm{~cm}^{-1}$. In most cases a difference of potential of 1,800 volts was applied; such a considerable field was necessary in view of the small mobility of ions in a dielectric liquid (mean value in hexane ${ }^{1}$ is $5 \times 10^{-4}$ cm./sec. per cm./volt).

The armature receiving the charge was connected with a quadrant electrometer of common pattern. It recorded the gradual charging of the system by the spontaneous current in the liquid. Bronson resistance was used for compensating this current. Bursts provoked sudden deviations of the electrometer needle and corresponding displacements on the curve of the record. It was possible easily to recognise bursts supplying more than $3 \times 10^{6}$ ions (the actual number of ions produced was considerably greater in consequence of the loss by recombination).

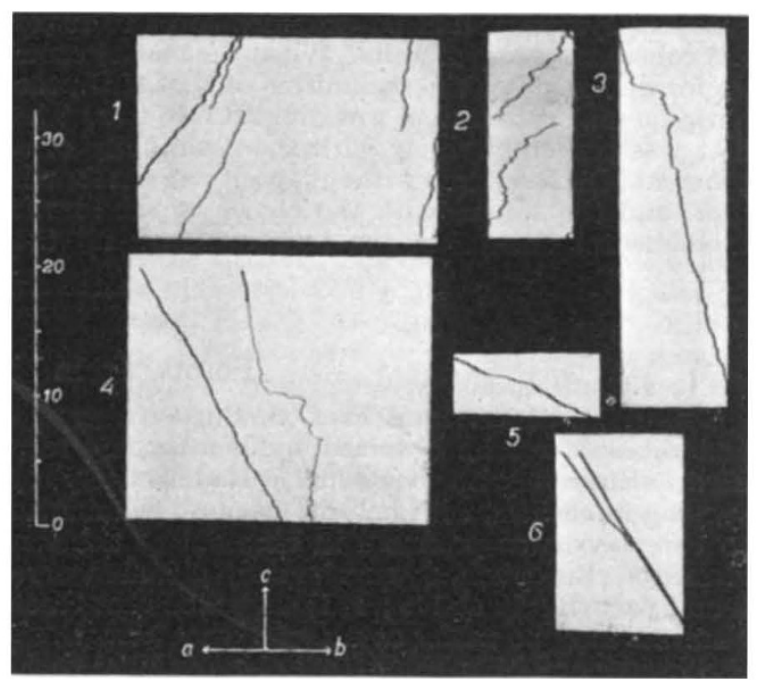

To characterise the phenomenon we give two numerical results and reproductions of some records obtained $^{2}$. In an unprotected ionisation chamber, the number of bursts was 2 in 11.7 hours. In the most favourable case, the chamber surrounded by lead blocks $5 \mathrm{~cm}$. thick and the aluminium cylinder put into the interior armature, the number of bursts increased to 37 in 13 hours.

In the accompanying illustration are reproduced the records of some typical bursts: Nos. 1, 2, 3 are simple bursts; Nos. 4, 5 seem to be double bursts ; No. 6 is of more complicated structure. The arrows indicate directions of deviations of the electrometer needle due to $(a)$ compensating currents, $(b)$ residual, and (c) the direction of movement of the sensitive film. On the left is a time-scale in minutes, which enables a rough estimate to be made of the duration of the bursts.

\section{Biarobrzeski.} I. ADAMCZEWSKI.

Institute of Theoretical Physics, Warsaw. May 28.

${ }^{1}$ One of us has recently made a detailed study of the mobility of ions in liquid paraffins: I. Adamczewski, Bull. Int. Acad. Polonaise. $217 ; 1934$. The existence in these liquids of three sorts of ions of different mobilities was discovered.

" More details will be given in a paper appearing in a forthcoming issue of Bull. Int. Acad. Polonaise. 